\title{
Hipertiroidismo, ictericia e hipertensión pulmonar
}

\author{
G. MOZO HERRERA, Ma.J. FERNÁNDEZ GONZÁLEZ, J. SALGADO BLANCO, \\ A. JIMENO CARRÚEZ
}

Servicio de Medicina Interna. Hospital Universitario. Valladolid

HYPERTHYROIDISM, JAUNDICE AND PULMONARY HYPERTEN SION

\section{RESUMEN}

Aunque la causa más frecuentemente asociada a la alteración de las pruebas de función hepática que presentan algunos pacientes hipertiroideos es la insuficiencia cardiaca concomitante, se han descrito casos sin insuficiencia cardiaca, con normalización de las pruebas hepáticas tras normalizar la función tiroidea.

También hay evidencia de enfermos hipertiroideos con hipertensión arterial pulmonar sin causa clara, regresando ésta a cifras normales, o descendiendo substancialmente, tras el tratamiento del hipertiroidismo.

Nosotros presentamos el caso de una mujer de 66 años con un hipertiroidismo debido a un bocio tóxico multinodular, con ictericia e hipertensión arterial pulmonar severa asociadas y no explicables por otras causas, regresando ambas con la normalización de la función tiroidea y sin evidencia de patología autoinmune asociada.

Posteriormente se comentan las explicaciones fisiopatológicas encontradas en la literatura sobre esta inusual asociación.

PALABRAS CLAVE: Hipertiroidismo. Ictericia. Hipertensión pulmonar.

\begin{abstract}
Although the most frecuent cause associated to the alteration of hepatic function tests that some hyperthyroid patients show is concomi tant heart insufficiency, some cases without heart insufficiency and nor malisation of hepatic tests after normalising the thyroid function have been described.

There is also some evidence of hyperthyroid patients with pulmonary arterial hypertensión, with no clear cause, coming back later to normal figures or substantially descending, after the treatment for hyperthyroi dism.

We show the concrete case of a 66 years woman, who has a due to toxic multinodular goiter hyperthyroidism with jaundice and serious pul monary hypertension associated, unexplainable by further causes, both regressing with normalisation of thyroid function with no evidence of associated autoimmune pathology.

Following, the physiopatology explanations about this unusual asso ciation found in literature, are commented.
\end{abstract}

KEY WORDS: Hyperthyroidism. Jaundice. Pulmonary hypertension.

Mozo Herrera G, Fernández González Ma.J, Salgado Blanco J, Jimeno Carrúez A. Hipertiroidismo, ictericia e hipertensión pulmonar. An Med Interna (Madrid) 2001; 18: 262-264.

\section{INTRODUCCIÓN}

Hasta un $76 \%$ de los pacientes hipertiroideos presentan anormalidades inespecíficas hepáticas, aunque son pocos los casos con alteraciones graves. La inmensa mayoría en relación con una insuficiencia cardiaca asociada al estado hiperti roideo (1), raramente secundaria a una hepatitis crónica activa autoinmune (pocos casos asocian hipertiroidismo y Cirrosis Biliar Primaria, asociación más frecuente con el hipotiroidismo) (2), y hay comunicados casos de hepatopatía en estados de tirotoxicosis en ausencia de insuficiencia cardiaca, con mejoría o curación de las alteraciones hepáticas tras recobrar el estado eutiroideo (3).
La incidencia de Hipertensión Pulmonar Primaria está incrementada en algunas enfermedades autoinmunes y en la cirrosis con hipertensión portal. Se han comunicado casos aislados de asociación con estados de hipertiroidismo (en su mayoría de etiología autoinmune) con mejoría sustancial de la hipertensión tras el tratamiento del hipertiroidismo, sugiriendo interacción entre la patología autoinmune, o entre las propias hormonas tiroideas con la etiología de la Hipertensión Pulmonar (4).

No conocemos ningún caso en la literatura que asocie ambos sucesos, alteraciones hepáticas en ausencia de insuficiencia cardiaca (en este caso hiperbilirrubinemia sin colostasis) e hipertensión pulmonar severa, en el seno de un estado

Trabajo aceptado: 30 de Septiembre de 1999

Correspondencia: G. Mozo Herrera. c/ Bolivia n ${ }^{\circ}$ 5, $6^{\circ} \mathrm{A}, 47014$ Valladolid. 
tirotóxico (de etiología no autoinmune), con mejoría de ambas alteraciones tras el tratamiento del cuadro de hipertiroidismo, como el que a continuación presentamos.

\section{CASO APORTADO}

Se trata de una mujer de 66 años de edad, con antecedentes de hipertiroidismo secundario a bocio multinodular hiperfuncionante diagnosticado tres años antes y tratado inicialmente con metimazol y posteriormente con varias sesiones de Yodo radiactivo, con mal control de la función tiroidea, que motivó varios ingresos en Cardiología por insuficiencia cardiaca mixta severa en estado de tirotoxicosis, descubriendo en uno de los ingresos una hipertensión pulmonar severa (presión sistólica en arteria pulmonar $65 \mathrm{mmHg}$ ) con insuficiencia tricúspide severa y sin otros datos de cardiopatía, que persistió en los mismos rangos tras superar el episodio de insuficiencia cardiaca, y tras descartar otras causas (gasometría, Rx tórax, espirometría, nuevos ecocardiogramas) se achacó a embolismos pulmonares de repetición, por lo que seguía únicamente tratamiento anticoagulante con dicumarínicos.

La paciente ingresa inicialmente a cargo del Servicio de Cirugía General al presentar cuadro de 15 días de evolución consistente en astenia importante, ictericia cutáneo-mucosa progresiva, pérdida de peso y diarrea de unas 4 deposiciones diarias. A la exploración física destacaba un estado caquéctico con atrofia y debilidad de la musculatura proximal exoftalmos, ictericia cutáneo-mucosa franca, bocio irregular, destacando un nódulo de unos $2 \mathrm{~cm}$, pétreo y adherido en lóbulo tiroideo izquierdo, temblor de fina oscilación distal con reflejos osteomusculares exaltados de forma difusa.

En los análisis básicos se observó una bilirrubina total de $7 \mathrm{mg} / \mathrm{dl}$, con una bilirrubina directa de $5,5 \mathrm{mg} / \mathrm{dl}$, con transaminasas, fosfatasa alcalina y GGT normales. La hemostasia presentaba un índice de protrombina del $41 \%$ (recordamos que estaba en tratamiento con dicumarínicos). En dos ecografías abdominales no se objetivó patología del parénquima hepático ni de las vías biliares intra y extrahepáticas, ni hepatomegalia u otros datos de congestión hepáti- ca. Igualmente se realizó una colangio pancreatografía retrógrada endoscopia, evidenciando un árbol biliar normal. Las serologías para virus de hepatitis fueron negativas.

En este momento se hace cargo de la paciente el Servicio de Medicina Interna, que tras evidenciar la clínica de tirotoxicosis, se confirma al detectar unos niveles de Tiroxina libre de $15,17 \mathrm{ng} / \mathrm{ml}$ (valores normales $0,73-2,01 \mathrm{ng} / \mathrm{ml}$ ), con TSH basal indetectable. Se realiza una PAAF del nódulo tiroideo, informada como adenoma folicular.Otras determinaciones realizadas fueron el estudio de autoanticuerpos (incluyendo los antitiroideos, antimicrosomales, TSI, antimitocondriales y antimúsculo liso), siendo todos ellos negativos.

Se inicia tratamiento con Metimazol, y previa analítica, que presenta valores de bilirrubinas y transaminasas similares a los previos, y de Ecocardiograma que confirma la persistencia de la hipertensión pulmonar en idénticos valores a los citados sin evidenciar cardiopatía que pueda justificarla, se realiza tiroidectomía total. La Anatomía Patológica de la pieza nos habla de una hiperplasia multinodular tiroidea con áreas de adenoma folicular.

La evolución de la paciente fue satisfactoria, normalizándose las cifras de bilirrubina a los siete días de la intervención, y objetivando a los dos meses un descenso de la PSAP a $45 \mathrm{mmHg}$, parámetros que se mantienen, junto con una buena situación clínica ocho meses después.

\section{DISCUSIÓN}

El caso presentado posee la peculiaridad de asociar dos alteraciones ciertamente poco comunes ya de forma aislada, la ictericia por hiperbilirrubinemia directa y la hipertensión pulmonar, de forma concomitante con un estado de hipertirotoxicosis.

Nuestra paciente no presentaba, al contrario que en anteriores ingresos, datos clínicos, semiología ni exploraciones complementarias sugerentes de insuficiencia cardiaca que justificara el cuadro de ictericia que motiva su ingreso, ni presentaba alteraciones de GGT ni fosfatasa alcalina que hicieran

\section{TABLA I}

CARACTERÍSTICAS DE NUESTRA PACIENTE, TERAPIA Y EVO LUCIÓ N (CO M PARADA CON OTROS CASOS DE LA LITERATURA)

\begin{tabular}{|c|c|c|c|c|c|}
\hline Enf. Tiroidea & Anticuerpos & $\begin{array}{l}\text { PAPs antes/después } \\
\text { del tratamiento } \\
(\mathrm{mmHa}) \text { meses }\end{array}$ & Tratamiento & $\begin{array}{l}\text { T4 libre antes/ después } \\
\text { del tratamiento (ng/ml) }\end{array}$ & $\begin{array}{l}\text { Bilirrubina antes/ despues } \\
\text { del tratamiento (mg/ } \mathrm{dl})\end{array}$ \\
\hline
\end{tabular}

sexo/ edad

transcurridos

\begin{tabular}{|c|c|c|c|c|c|}
\hline $\mathrm{M} / 66 \mathrm{bmn}$ & negativos & $65 / 45,2$ & cirugía & $15,17 / 1,5$ & $7 / 0,8$ \\
\hline \multicolumn{6}{|c|}{$\begin{array}{l}\text { O tros casos comunicados por distintos autores: } \\
\text { (№ Ref.) }\end{array}$} \\
\hline $\begin{array}{l}\text { (1) primaria } \\
\mathrm{H} / 42\end{array}$ & negativos & no hipertensión & iodo 31 & $\begin{array}{l}\text { T4 total: } 19,4 / 9,2 \\
(\mu \mathrm{g} / 100 \mathrm{ml})\end{array}$ & $16,7 /$ normal \\
\hline $\begin{array}{l}\text { (4) Graves } \\
\text { M/54 }\end{array}$ & $\begin{array}{c}\text { TSI, } \\
\text { antimicro- } \\
\text { somales }\end{array}$ & $56 / 35,1$ & $\begin{array}{l}\text { propil- } \\
\text { tiouracilo }\end{array}$ & $6,14 / 0,66$ & no ictericia \\
\hline $\begin{array}{l}\text { (4) Graves } \\
\mathrm{H} / 24\end{array}$ & TSI & $33 / 21,12$ & tiomazol & $8,5 / 0,9$ & no ictericia \\
\hline $\begin{array}{l}\text { (8) Graves } \\
M / 28\end{array}$ & $\begin{array}{c}\text { TSI, } \\
\text { antimicro- } \\
\text { somales }\end{array}$ & $62 / 45,1$ & neocarbimazol & 3,3 / normal & no ictericia \\
\hline
\end{tabular}

PAPS: Presión en Arteria Pulmonar sistólica. bmn: bocio multi nodular. $\mathrm{M}$ : mujer. H: hombre. TSI: inmunoglobulina estimulante del tiroides. T4: tiroxina (rango T4 libre: 0,73-2,01 ng/ml, rango T4 total: 4,5-11 mg/100ml). № Ref.: referencia bibliográfica citada. 
pensar en colostasis. El hecho de la normalidad de las transaminasas excluye cuadros de citolisis hepática, por lo que no se consideró conveniente la realización de biopsia hepática. Las pruebas de imagen. ecografías y CPRE descartaron alteraciones de las vías biliares. También se eliminaron otras causas de ictericia, como hepatotoxicidad y patología infecciosa o autoinmune.

Tras estas evidencias, y a la vista de la evolución hacia la normalización de la bilirrubina después de superar la situación de hipertiroidismo, nos hace pensar en el propio estado de tirotoxicosis, como causante de la hiperbilirrubinemia de la paciente.

Otros autores han encontrado cuadros similares con grados variables de citolisis y colostasis, con evolución satisfactoria después de la normalización de la función tiroidea $(1,3,5)$, hepatitis granulomatosa de forma concomitante con hipertiroidismo por enf de Graves con buena evolución clínica tras tratamiento antitiroideo,o mejoría de hepatopatías preexistentes una vez instaurado el tratamiento antitiroideo (2).

Se han propuesto por ello varias teorías para explicar esta curiosa asociación, una vez descartada la insuficiencia cardiaca, (que sí es una causa conocida de alteraciones hepáticas en el seno de un hipertiroidismo) $(6,7)$. Johnston supone que el estado hipermetabólico podría incrementar el consumo de oxígeno por el hepatocito, sin aumentar de forma paralela el flujo sanguíneo, acentuándose la baja presión de oxígeno en las zonas centrolobulare, lo que causaría disfunción con colestasis de estos hepatocitos, también se ha propuesto un efecto tóxico directo sobre las células hepáticas de la propia tiroxina (1).

Respecto a la hipertensión pulmonar, ya evidenciada y mantenida en nuestra enferma desde dos años antes al actual ingreso, y habiéndose descartado otras causas de Hipertensión Pulmonar más comunes, nos hace suponer, a la vista de la evolución tras la normalización de la función tiroidea, que el hipertiroidismo ha jugado un claro papel etiológico y en efecto varios autores han comunicado casos de hipertensión pul- monar (sin otra causa aparente) de forma concomitante con tirotoxicosis bien de etiología autoinmune (presencia de anticuerpos antitiroideos, antimicrosomales y/o TSI positivos), con buena respuesta de la primera tras el tratamiento de la segunda $(4,8)$, o no autoinmune como nuestra paciente, como un caso de hipertiroidismo por bocio multinodular e HT Pulmonar, con descenso de la PSAP tras la normalización de la función tiroidea (4).

La asociación de hipertensión pulmonar e ictericia con un cuadro de hipertiroidismo, como en nuestro caso, es un hecho francamente raro y no esperable en la clínica diaria, pero si sería conveniente, a la vista de los múltiples casos comunicados, y a pesar de no estar contemplado así en los manuales de manejo más común, considerar el estado hipertiroideo en casos de ictericia o alteración de pruebas de función hepática y en casos de hipertensión pulmonar en los que no se evidencie una causa más común tras la realización de los estudios convenientes.

Hay una evidencia clara de efectos directos de las hormonas tiroideas sobre el miocardio, sumándose a otros factores indirectos (sistema nervioso simpático, alteración de la circulación periférica, metabolismo energético). El hipertiroidismo propicia un aumento del flujo sanguíneo y del gasto cardiaco. Situaciones que alteren la relación flujo sanguíneo/resistencia del lecho vascular pulmonar pueden potencialmente provocar una hipertensión pulmonar.

Aunque no están claramente estudiadas las relaciones entre hipertiroidismo e hipertensión pulmonar, se proponen varios mecanismos patofisiológicos que podrían explicar el hallazgo de una hipertensión pulmonar en los pacientes con hipertiroidismo: el agravamiento de una hipertensión pulmonar subclínica preexistente, un gasto cardiaco incrementado (en sujetos sanos esto no produce hipertensión pulmonar), y, más probablemente, unas resistencias vasculares pulmonares elevadas secundarias a una adaptación anormal en presencia de un aumento del flujo sanguíneo $(4,8)$.

\section{Bibliografía}

1. Joseph, Yao, John B, Gross, Jurgen Ludwig, Don C Purnell. Cholestatic Jaundice in Hyperthyroidism. Am J Med 1989; 86: 619-20.

2. Nick P Thompson, Serena Leader, Crawford P Jamieson, W Rodney Burnham, Andrew K Burroughs. Reversible Jaundice in Primary Biliary Cirrhosis Due to Hyperthyroidism. Gastroenterology 1994; 106: 1342-43.

3. Fong, McHutchison, Reynolds. Hyperthyroidism and hepatic dysfunction. A case series analysis. J Clin Gastroenterol 1992; 14 (3): 240-4.

4. R Thurnheer, R Jenni, EW Russi, P Greminger, R Speich. Hyperthyroidism and pulmonary hypertension. J Intern Med 1997; 242: 185-8.

5. Cathebras PJ, Mosnier JF, Gouilloud S, Bouchou K, Rousset H. Hepatic granulomatosis in a patient with Graves' disease. Eur J Gastroenterol Hepatol 1995; 7 (9): 905-8.

6. Fischer MR, Spes $\mathrm{CH}$, Huss R, Gartner R. Immunogenic hyperthyroidism with hyperdynamic heart failure and early cirrhotic transformation of the liver. Dtsch Med Wochenschr 1997; 122 (11): 323-7.

7. Lee TM, Kuo SH, Lee YT. Case report: reversible systolic heart failure and deep jaundice in hyperthyroidism. Am J Med Sci 1996; 312 (5): 246-8.

8. R Alcázar, M Rey, P de Sequera, R Alegre, A Rovira, C Caramelo. Reversibilidad de hipertensión pulmonar asociada a hipertiroidismo autoinmune. Rev Esp Cardiol 1995; 48 (2): 142-4. 